\title{
MÉTODO PARA CORREÇÃO DE IMAGENS DE PHANTOM E CLASSIFICAÇÃO DE SUAS ESTRUTURAS DE INTERESSE
}

\author{
Bruno Barufaldi \\ Departamento de Informática, Universidade Federal da Paraíba, João Pessoa, Brasil. \\ barufaldi@di.ufpb.br \\ Amanda B. Cavalcanti \\ Departamento de Informática, Universidade Federal da Paraíba, João Pessoa, Brasil. \\ amanda@di.ufpb.br \\ Leonardo V. Batista \\ Departamento de Informática, Universidade Federal da Paraíba, João Pessoa, Brasil. \\ leonardo@di.ufpb.br \\ Renata F. Góis \\ Departamento de Engenharia Elétrica, Universidade de São Paulo, São Carlos, Brasil. \\ rfgoes@sc.usp.br \\ Homero Schiabel \\ Departamento de Engenharia Elétrica, Universidade de São Paulo, São Carlos, Brasil. \\ homero@sc.usp.br \\ José F. G. Carvalho \\ Governo da Paraíba - Agência Estadual de Vigilância Sanitária da Paraíba \\ João Pessoa, PB, Brasil.
}

\section{RESUMO}

O controle de câncer de mama representa um dos grandes desafios que os serviços de saúde pública enfrentam atualmente. Para realizar o controle de qualidade de sistemas mamográficos o Ministério da Saúde exige o uso de simuladores radiográficos (phantoms) de mama. Com o objetivo de reduzir a subjetividade na avaliação das imagens de phantom pela inspeção visual humana, está sendo desenvolvido um sistema computadorizado que utiliza um método de correção em imagens digitalizadas, associado à classificação de suas estruturas de interesse pelo critério de visibilidade. Ao comparar os resultados da classificação através do algoritmo J48 da ferramenta WEKA com e sem a correção das imagens, essa técnica apresentou uma melhora significativa na eficácia para determinadas estruturas do phantom.

PALAVRAS-CHAVE: câncer de mama, simulador radiográfico, phantom MAMA-CDM, correção de imagens digitalizadas, controle de qualidade, percepção visual humana.

\section{CORRETION METHOD OF PHANTOM IMAGES AND CLASSIFICATION OF THEIR STRUCTURES OF INTEREST}

\begin{abstract}
Breast cancer control represents one of the greatest challenges that public health service faces nowadays. To execute the quality control of mammographic systems, the Brazilian Health Ministry demands the use of breast phantoms. Aiming to reduce the subjectivity present in the evaluation of phantom images through human visual inspection, a computerised system has been developed that uses a correction method in its digitised
\end{abstract}


images, associated with the classification of its structures of interest by the visibility criterion. Comparing the results of the classification using the J48 algorithm of the WEKA package with and without image correction, this method presented a significant improvement in the effectiveness for determining structures of the phantom.

KEY-WORDS: breast cancer, phantom MAMA-CDM, quality control, digitised image correction, visual perception.

\section{MÉTODO PARA CORREÇÃO DE IMAGENS DE PHANTOM E CLASSIFICAÇÃO DE SUAS ESTRUTURAS DE INTERESSE}

\section{INTRODUÇ̃̃O}

O câncer de mama é a segunda neoplasia mais incidente na população mundial feminina. A cada ano, cerca de $22 \%$ dos casos novos de câncer em mulheres são de mama (INCA, 2009). De acordo com os dados da GLOBOCAN (International Agency for Research on Cancer), em 2008 foram registrados em todo o mundo 691 mil novos casos de câncer de mama em países em desenvolvimento e 692 mil em países desenvolvidos. No entanto, o número total de mortes pela doença em países em desenvolvimento foi de $268 \mathrm{mil}$, em contraste com 189 mil mortes nos países desenvolvidos (TIEZZI, 2010).

A mamografia é o exame mais confiável e acessível para o rastreamento do câncer de mama. A sua eficácia e indicação são aceitas mundialmente e, na faixa etária entre 50 e 69 anos, a redução da mortalidade pela doença é da ordem de $30 \%$. Já o benefício para mulheres entre 40 a 50 anos gira em torno de 15 a 18\% (TIEZZI, 2010).

O mecanismo de inspeção das mamografias é um procedimento custoso e predisposto a erros. Para a redução destas falhas, o Programa de Certificação da Qualidade em Mamografia do Colégio Brasileiro de Radiologia (CBR) exige que os serviços de mamografia efetuem o registro radiográfico de um simulador de mama, denominado phantoms (CINTI et al., 2004).

A digitalização de imagens radiográficas é uma etapa importante para processo de detecção das estruturas de interesse para o diagnóstico médico. O método mais simples para a aquisição de uma imagem no formato digital é através da utilização de um scanner. Para que os esquemas computacionais tenham sucesso, a digitalização deve seguir alguns critérios mínimos quanto à relação sinal ruído da imagem e do dispositivo digitalizador utilizado.

A utilização de sistemas computadorizados de auxílio ao diagnóstico torna-se necessária para manter a qualidade das atividades e serviços clínicos (HOGGE et al., 1999). O objetivo principal da produção desses sistemas está em tentar eliminar a subjetividade na análise especialista, devida às limitações próprias do ser humano, diminuindo substancialmente a carga de trabalho e saturação dos radiologistas.

Atualmente existem diversas aplicações na área de Inteligência Artificial utilizadas na Medicina. Entre as mais conhecidas, estão os sistemas de apoio ao diagnóstico médico. Para o computador elaborar uma hipótese diagnóstica, é necessário que o especialista 
informe todos os dados relevantes observados. Com isso, o sistema é capaz de processar essas informações de maneira eficiente, auxiliando a elaboração do diagnóstico.

O objetivo deste trabalho é desenvolver um subsistema do SIstema de Auxílio ao Diagnóstico médico (SIAD), denominado QualiMamo, voltado para automatização da maior parte das etapas do processo de controle de qualidade de mamógrafos atualmente adotado pela Agência de Vigilância Sanitária do Estado da Paraíba (AGEVISA-PB), visando ser uma ferramenta de auxílio ao controle de qualidade. Esse subsistema busca reduzir a subjetividade na avaliação das imagens do phantom MAMA-CDM (MEDEIROS et al., 2002) pela inspeção visual humana, utilizando um método de correção em imagens digitalizadas, associado à classificação de suas estruturas de interesse pelo critério de visibilidade.

\section{FUNDAMENTAÇÃO TEÓRICA}

Essa seção apresenta os conceitos considerados relevantes à completa compreensão deste trabalho.

\subsection{FILMES RADIOGRÁFICOS}

O principal objetivo da realização de qualquer tipo de tomada radiográfica está na fiel reprodução dos objetos de interesse para o diagnóstico. Para a representação radiográfica é utilizado um filme sensível à luz juntamente a uma tela florescente (écran), capaz de converter cada fóton de raios $\mathrm{X}$ em um feixe de fótons de luz visível que é absorvido pelo filme. Para descrever a resposta de um sistema écran-filme à exposição radiográfica utiliza-se um gráfico chamado curva característica, que relaciona a exposição radiográfica com a densidade ótica produzida no filme. A resposta de um filme ou de uma combinação écran-filme à exposição de raios $\mathrm{X}$ é dada pela curva característica, que usa uma escala logarítmica para representar a exposição.

Por intermédio da curva característica pode-se determinar o contraste do filme, através do grau de inclinação da parte linear da curva, ou seja, da faixa de densidades úteis. Uma inclinação mais acentuada indica alto contraste.

\subsection{PROCESSO DE DIGITALIZAÇÃO}

Os aspectos de digitalização de filmes radiográficos devem ser observados para garantir a eficiência do processo do diagnóstico. Os níveis de cinza vão ser processados a fim de informarem o tamanho e localização das estruturas de interesse da imagem. Se a digitalização não fornecer um tamanho suficientemente pequeno de pixel, algumas estruturas pequenas podem ser desprezadas e até mesmo desaparecer (CHAN et al., 2004).

Muitos equipamentos radiográficos digitais têm sido desenvolvidos para a realização do diagnóstico, mas a aceitação clínica tem sido lenta, devido ao seu alto custo. Tendo em vista as vantagens de se adquirir a imagem radiográfica em sua forma digital, a opção imediata é adquiri-la por meio da digitalização utilizando um scanner que contenha um sistema adaptador de filmes, onde se registra a luz transmitida e não a refletida.

No entanto, sabe-se que o processo de digitalização da imagem radiográfica gera ruídos na imagem que correspondem a distorções visuais que não contribuem com informação, muitas vezes dificultando a visualização das estruturas de interesse das imagens (GONZALEZ-LÓPEZ, 2007). 
Sendo assim, é necessário realizar um acompanhamento do desempenho do digitalizador utilizado, verificando a confiabilidade da resposta dada por este sistema bem como a qualidade de suas imagens.

\subsection{SIMULADOR RADIOGRÁFICO MAMA-CDM}

Nos centros de radiodiagnósticos são utilizados rotineiramente simuladores radiográficos (ou phantoms) que contêm estruturas que representam os tecidos da mama. O principal objetivo dos phantoms é simular a interação do feixe de raios $\mathrm{X}$ com o paciente, para avaliar a atenuação da radiação e qualidade da imagem no filme mamográfico.

O phantom MAMA-CDM simula uma mama comprimida entre $4 \mathrm{~cm}$ e $5 \mathrm{~cm}$ e possui detalhes que produzem imagens radiográficas semelhantes às estruturas presentes na mama (microcalcificações, discos, fibras e massas tumorais).

São avaliados nas imagens dos simuladores a definição (resolução espacial), os detalhes de alto contraste (microcalcificações), os limiares de baixo contraste (discos), os detalhes lineares de baixo contraste (tecido fibroso), as massas tumorais e a densidade ótica de fundo. A Figura 1 apresenta uma foto do phantom MAMA-CDM e sua imagem radiográfica.
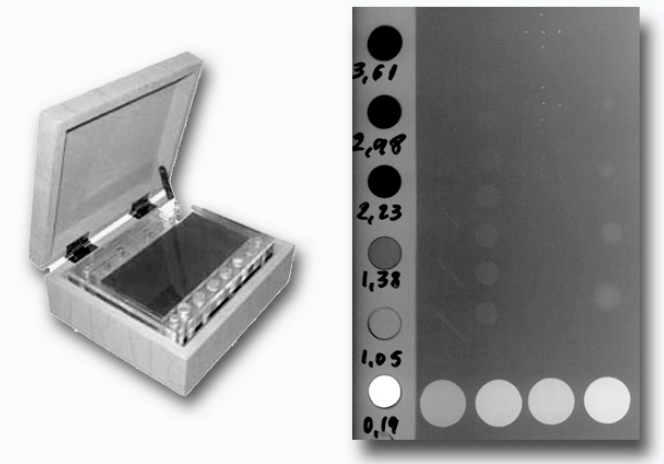

Figura 1 - Simulador radiográfico MAMA-CDM e sua imagem radiográfica.

\subsection{WEKA}

O pacote WEKA (Waikato Environment for Knowledge Analysis) é formando por um conjunto de implementações de algoritmos utilizados em Mineração de Dados. Esse pacote inclui uma variedade de métodos de aprendizagem para o conjunto de dados a ser avaliado, denominados classificadores.

As árvores de decisão têm diversas vantagens quando comparadas a outras técnicas. A principal está em sua simplicidade e facilidade na interpretação dos resultados. O J48 do WEKA é a implementação otimizada do algoritmo de construção de árvores de decisão C4.5, que foi a última versão pública do algoritmo (WITTEN e FRANK, 2005). Esse algoritmo constrói um modelo de árvore de decisão baseado em um conjunto de dados de treinamento e usa esse modelo para classificar as instâncias de um conjunto de testes. 


\section{MATERIAIS E MÉTODOS}

Uma das abordagens de inspeção visual do filme radiográfico consiste em determinar a visibilidade das estruturas de interesse presentes na imagem do phantom MAMA-CDM.

Para a obtenção das imagens digitalizadas do phantom, utilizou-se o scanner Microtek, modelo ScanMaker i800, e foram digitalizadas em níveis de cinza, com 16 bits de resolução de contraste e 1200 x 1200 dpi de resolução espacial. Para a produção da curva característica do filme foi utilizado um filme radiográfico da marca $A g f a$, relevado por uma processadora de marca Microtek-MX-2.

A eficácia do sistema foi aferida utilizando 100 imagens corrigidas (conforme descrito na seção 3.2) do simulador radiográfico, com um total de 1900 estruturas analisadas entre discos de baixo contraste, fibras e massas tumorais. O comportamento do software é visualizado através das curvas ROC (Receiver Operating Characteristic), em que são verificadas a sensibilidade e a especificidade do sistema, à medida que se reduz o contraste das estruturas.

\subsection{CURVA CARACTERÍSTICA}

Para a produção da curva característica do filme foram necessários um sensitômetro e um densitômetro (Mod. X-Rite 334) calibrados para medir as densidades óticas através de uma varredura sobre a região do filme sensibilizado com 21 passos de exposição. Com a utilização desses equipamentos, foi possível determinar a relação entre as densidades óticas e a exposição do filme. Assim, as densidades óticas correspondentes a cada exposição diferente possibilitaram o levantamento da curva característica.

Uma vez definida a curva característica do filme, foi necessário conhecer a curva característica do digitalizador das imagens dos phantoms (Microtek ScanMaker i800). Para isso, digitalizou-se dez vezes uma mesma imagem do filme neste mesmo digitalizador em diferentes posições, o que permitiu identificar as variações de intensidade existentes na escala produzidas pelo sensitômetro, correspondentes aos 21 passos de exposição do filme.

A construção da curva característica do digitalizador depende da relação entre o nível de cinza dos pontos de exposição e as medidas das densidades óticas. Essa relação associa a média dos valores dos pixels de cada região de interesse com o valor de densidade que tenha o mesmo passo de exposição. Desta forma, foi possível analisar a variação dos valores de pixels de uma região para diferentes digitalizações e, assim, verificar se há variação significativa nos pixels das imagens de um filme digitalizado em diferentes posições do digitalizador. A Figura 2 apresenta um exemplo de uma curva característica de um filme. 

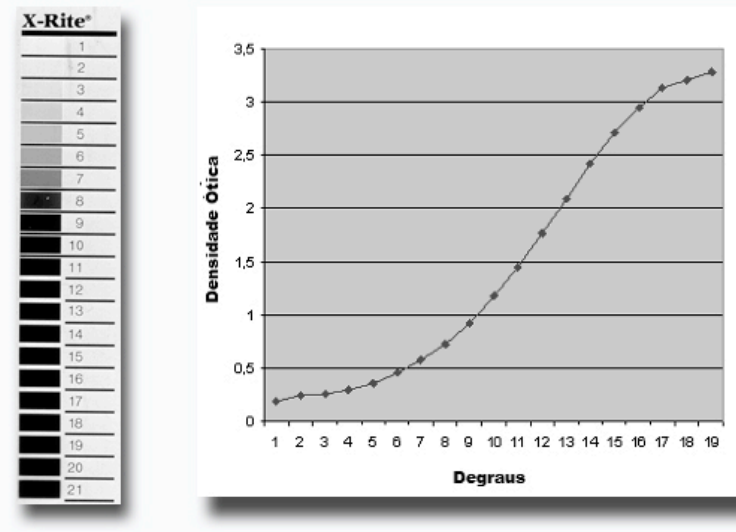

Figura 2 - Exemplo de gráfico da curva característica de um filme radiográfico.

\subsection{CORREÇÃO DAS IMAGENS}

Conhecida a curva característica do scanner Microtek, foi possível realizar a correção das imagens digitalizadas. O procedimento tem como função calcular, através de interpolação matemática, o polinômio que melhor representa os pontos amostrados referentes às densidades óticas obtidas para cada digitalizador. Esse polinômio tem como objetivo corrigir os níveis de cinza da imagem de modo a torná-los similares aos das imagens tomadas como referências.

Após determinado o polinômio, é necessário aplicá-lo às imagens do simulador radiográfico que foram digitalizadas. Para isso, foram realizadas operações pontuais na região que contém as estruturas de interesse da imagem. A partir desse pré-processamento, foi possível compensar o desvio do digitalizador no que se refere à intensidade da imagem e, posteriormente, localizar e classificar as estruturas da imagem resultante.

\subsection{CLASSIFICAÇÃO COM WEKA}

Para realizar a etapa de classificação, é necessário detectar as estruturas de interesse das imagens dos phantoms utilizados. As estruturas são detectadas através de uma técnica baseada em casamento por correlação (GONZALES e WOODS, 2002) apresentada em trabalhos anteriores pelos autores deste trabalho (BARUFALDI et al., 2009). Com o sistema desenvolvido é possível localizar as estruturas de interesse com precisão.

Para a construção dos modelos de classificação que serão gerados pelo algoritmo J48, é necessário pré-selecionar alguns atributos da imagem para realização da etapa de treinamento do sistema. É importante destacar que nem todos esses atributos serão empregados para a classificação das estruturas, já que somente algumas das características da imagem são consideradas relevantes pelo algoritmo.

Os atributos utilizados na etapa de treinamento do sistema foram determinados através do seletor de atributos da ferramenta WEKA. Esses atributos podem ter natureza global, em relação à imagem, ou local, referentes às estruturas de interesse detectadas pelo sistema.

A Tabela 1 define os atributos globais utilizados para realização da etapa de treinamento do sistema. Esses atributos serão extraídos de cada região de interesse da imagem, onde $p(i, j)$ é o nível de cinza do pixel na posição $(i, j)$ e $w$ e $h$ são, respectivamente, a largura e altura da imagem. 
Tabela 1: Atributos globais extraídos das imagens dos phantoms.

\begin{tabular}{|c|c|c|c|c|c|}
\hline Atributo & $\begin{array}{c}\text { Média dos pixels da } \\
\text { imagem }\end{array}$ & $\begin{array}{c}\text { Média do módulo da diferença } \\
\text { entre os pixels da imagem e a } \\
\text { média da imagem }\end{array}$ & Variância da imagem & $\begin{array}{c}\text { Desvio } \\
\text { Padrão da } \\
\text { imagem }\end{array}$ & $\begin{array}{c}\text { Moda dos pixels } \\
\text { da imagem }\end{array}$ \\
\hline Equação & $\mu=\sum_{i=1}^{w} \sum_{j=1}^{h} \frac{p(i, j)}{\left(w^{*} h\right)}$ & $x=\sum_{i=1}^{w} \sum_{j=1}^{h} \frac{|p(i, j)-\mu|}{\left(w^{*} h\right)}$ & $v=\sum_{i=1}^{w} \sum_{j=1}^{h} \frac{(p(i, j)-\mu)^{2}}{\left(w^{*} h\right)}$ & $\sigma=\sqrt{v}$ & $\begin{array}{c}\text { Nível de cinza } \\
\text { mais frequente } \\
\text { em } p(i, j)\end{array}$ \\
\hline
\end{tabular}

A Tabela 2 apresenta os atributos extraídos de cada estrutura também utilizados na etapa de treinamento, onde $p_{e}(i, j)$ e $p_{b}(i, j)$ são os níveis de cinza dos pixels da região interna (estrutura) e externa (plano de fundo) do filtro de tamanho $w^{*} h$ na posição $(i, j)$ da imagem.

Tabela 2: Atributos extraídos das imagens dos phantoms após a detecção das estruturas de interesse.

\begin{tabular}{|c|c|c|c|c|}
\hline Atributo & $\begin{array}{c}\text { Média dos pixels da } \\
\text { estrutura }\end{array}$ & $\begin{array}{c}\text { Média dos pixels do } \\
\text { plano de fundo }\end{array}$ & $\begin{array}{c}\text { Diferença das médias dos } \\
\text { níveis de cinza dos pixels da } \\
\text { estrutura e plano de fundo }\end{array}$ & $\begin{array}{c}\text { Razão de Weber } \\
\text { (Gonzales e Woods, } \\
\text { 2002) }\end{array}$ \\
\hline Equação & $\mu_{e}=\sum_{i=1}^{w} \sum_{j=1}^{h} \frac{p_{e}(i, j)}{\left(w^{*} h\right)}$ & $\mu_{b}=\sum_{i=1}^{w} \sum_{j=1}^{h} \frac{p_{b}(i, j)}{w^{*} h}$ & $\Delta \mu=\mu_{e}-\mu_{b}$ & $W=\frac{\Delta \mu}{\mu_{e}}$ \\
\hline
\end{tabular}

A etapa de treinamento consiste em produzir, através do algoritmo J48 do pacote WEKA, um modelo de árvore de decisão para cada estrutura de interesse, que permanecerá estático durante o processamento das imagens pelo sistema na etapa de classificação. Para tanto, utilizou-se 50 imagens laudadas por especialistas do Programa de Controle de Qualidade de Mamógrafos da AGEVISA-PB.

Um total de 300 estruturas foi extraído dessas imagens para treinamento, contendo discos de baixo contraste, fibras e massas tumorais. Essas estruturas foram classificadas por especialistas de acordo com sua visibilidade, ou seja, se são visíveis ou não. É importante destacar que os laudos das imagens foram feitos por dois ou mais técnicos especializados, de maneira a diminuir a subjetividade própria da inspeção visual e aumentar a consistência do sistema.

Nessa etapa são criados os arquivos do formato padrão $A R F F$ do WEKA, com todos os dados de entrada citados anteriormente. É possível selecionar os atributos relevantes para o treinamento através da própria ferramenta, na etapa de pré-processamento.

$\mathrm{Na}$ etapa seguinte, de classificação, foram implementadas árvores de decisão a partir dos modelos produzidos na etapa anterior. Foram utilizadas 100 imagens de simulador radiográfico laudadas para classificação, com um total de 1900 estruturas. É importante destacar que as imagens processadas na etapa de classificação são distintas das de treinamento.

O comportamento do software é visualizado através de curvas ROC para cada estrutura de interesse, em que são verificadas a sensibilidade e a especificidade do sistema. Para a construção das curvas ROC o valor do atributo $\Delta \mu$ foi alterado dentro de um certo intervalo, uma vez que este foi o atributo de maior relevância nas etapas de treinamento de todas as estruturas de interesse. A escolha desse atributo se deu por este ter sido o de maior relevância nas etapas de treinamento de todas as estruturas de interesse. 


\section{RESULTADOS}

A Figura 3 apresenta os gráficos da curva característica do filme e do scanner Microtek ScanMaker i800, juntamente com seu ajuste polinomial filme/scanner.

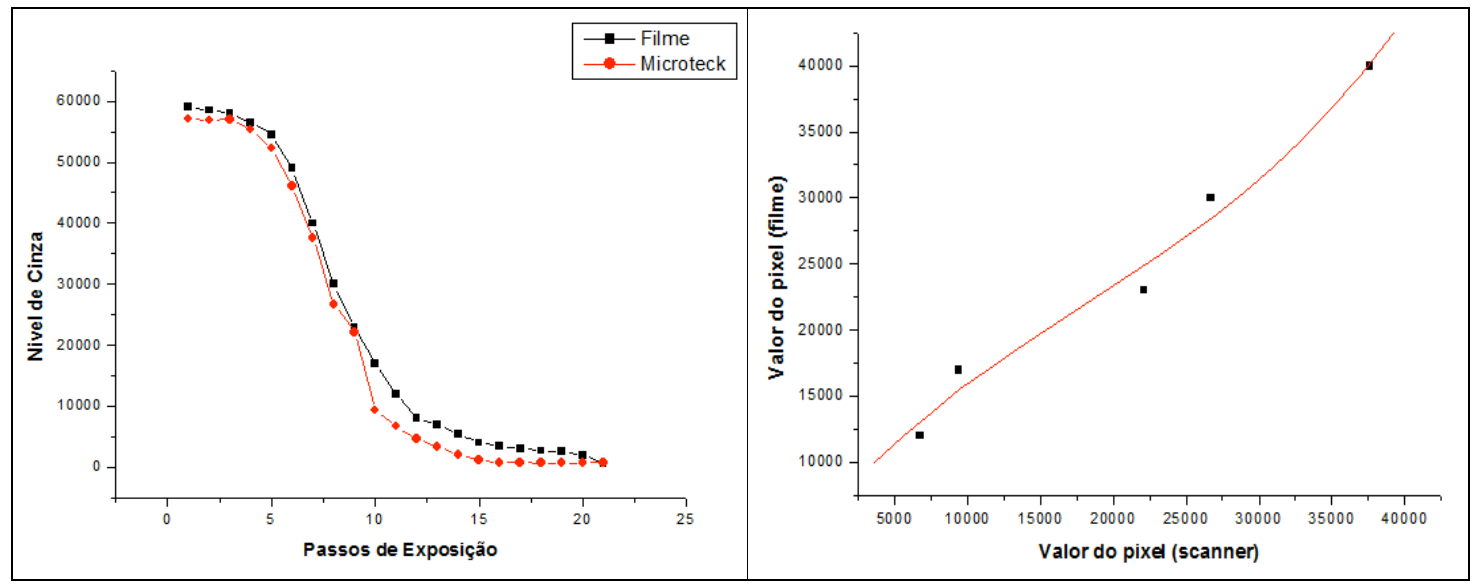

Figura 3. Curva característica filme/scanner e ajuste polinomial de grau 3 filme/scanner, $f(x)=5,76 E-10 * x^{3}-3,13 E-5 * x^{2}+1,28 * x+5689$.

Na comparação das imagens da Figura 4, nota-se que quando aplicado o polinômio de correção, as imagens digitalizadas apresentaram mais fidelidade às densidades óticas reais do filme.
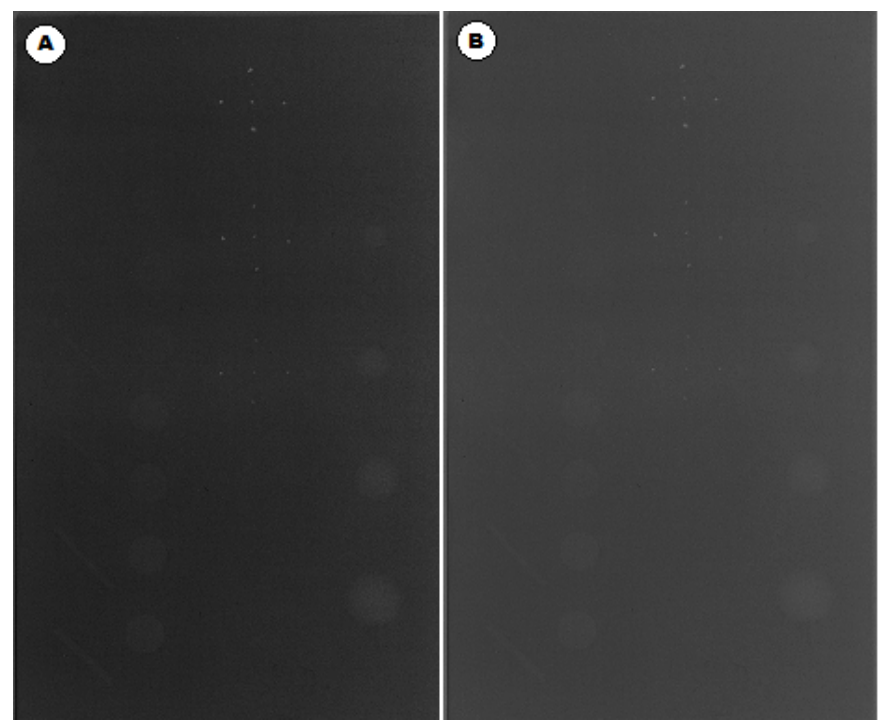

Figura 1 - (A) Imagem do simulador radiográfico original digitalizada no scanner Microteck ScanMaker i800 e (B) resultado da aplicação do polinômio de correção na mesma imagem.

Para representação da eficácia do sistema, são apresentadas as tabelas de contingência (ou matrizes de confusão), que indicam as taxas de acerto da classificação. A Tabela 3 mostra as matrizes de confusão das massas, discos e fibras, classificadas de acordo com o modelo gerado pelo algoritmo J48 com e sem a correção. 
Tabela 3: Tabela de contingência das estruturas após a etapa de classificação.

\begin{tabular}{|c|c|c|c|c|c|c|c|}
\hline & & \multicolumn{2}{|c|}{$\begin{array}{c}\text { Classificação das } \\
\text { fibras sem correção }\end{array}$} & \multicolumn{2}{|c|}{$\begin{array}{l}\text { Classificação das } \\
\text { massas sem correção }\end{array}$} & \multicolumn{2}{|c|}{$\begin{array}{c}\text { Classificação dos } \\
\text { discos sem correção }\end{array}$} \\
\hline & & Visível & Não Visível & Visível & Não Visível & Visível & Não Visível \\
\hline \multirow{2}{*}{ 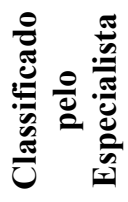 } & $\sum_{\substack{\infty \\
\infty}}^{\bar{D}}$ & 0,963 & $\mathbf{0 , 0 3 7}$ & 0,915 & 0,085 & 0,922 & 0,078 \\
\hline & 漓 & 0,593 & 0,407 & 0,04 & 0,96 & 0,11 & 0,89 \\
\hline & & \multicolumn{2}{|c|}{$\begin{array}{l}\text { Classificação das } \\
\text { fibras com correção }\end{array}$} & \multicolumn{2}{|c|}{$\begin{array}{l}\text { Classificação das } \\
\text { massas com correção }\end{array}$} & \multicolumn{2}{|c|}{$\begin{array}{l}\text { Classificação dos } \\
\text { discos com correção }\end{array}$} \\
\hline & & Visível & Não Visível & Visível & Não Visível & Visível & Não Visível \\
\hline \multirow{2}{*}{ 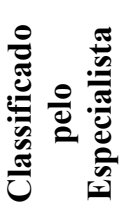 } & $\sum_{\substack{\infty \\
>}}^{\bar{D}}$ & 0,954 & 0,046 & 0,915 & 0,085 & 0,922 & 0,078 \\
\hline & 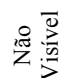 & 0,407 & 0,593 & 0,04 & 0,96 & 0,11 & 0,89 \\
\hline
\end{tabular}

A Tabela 4 apresenta as taxas de preditividade positiva e negativa, sensibilidade, especificidade, eficiência e acurácia do sistema, para a classificação de cada estrutura de interesse.

Tabela 4: Medidas de eficácia do software para classificação das estruturas utilizando o algoritmo $\mathbf{J 4 8}$.

\begin{tabular}{|l|c|c|c|c|c|c|}
\hline & \multicolumn{3}{|c|}{$\begin{array}{c}\text { Classificação das estruturas } \\
\text { sem correção }\end{array}$} & \multicolumn{3}{c|}{$\begin{array}{c}\text { Classificação das } \\
\text { estruturas com correção }\end{array}$} \\
\hline & Massas & Discos & Fibras & Massas & Discos & Fibras \\
\hline Acurácia & 0,924 & 0,915 & $\mathbf{0 , 8 3 3}$ & 0,926 & 0,915 & $\mathbf{0 , 8 7 0}$ \\
\hline Sensibilidade & 0,989 & 0,968 & $\mathbf{0 , 8 4 4}$ & 0,990 & 0,968 & $\mathbf{0 , 8 8 7}$ \\
\hline Especificidade & 0,738 & 0,758 & $\mathbf{0 , 7 6 0}$ & 0,734 & 0,758 & $\mathbf{0 , 7 9 0}$ \\
\hline Eficiência & 0,864 & 0,863 & $\mathbf{0 , 8 0 2}$ & 0,866 & 0,863 & $\mathbf{0 , 8 3 9}$ \\
\hline Preditividade Positiva & 0,915 & 0,922 & $\mathbf{0 , 9 6 1}$ & 0,912 & 0,922 & $\mathbf{0 , 9 5 2}$ \\
\hline Preditividade Negativa & 0,960 & 0,890 & $\mathbf{0 , 4 1 0}$ & 0,980 & 0,890 & $\mathbf{0 , 5 9 7}$ \\
\hline
\end{tabular}

O comportamento do sistema pode ser verificado através das curvas ROC, conforme apresentado na Figura 5. 


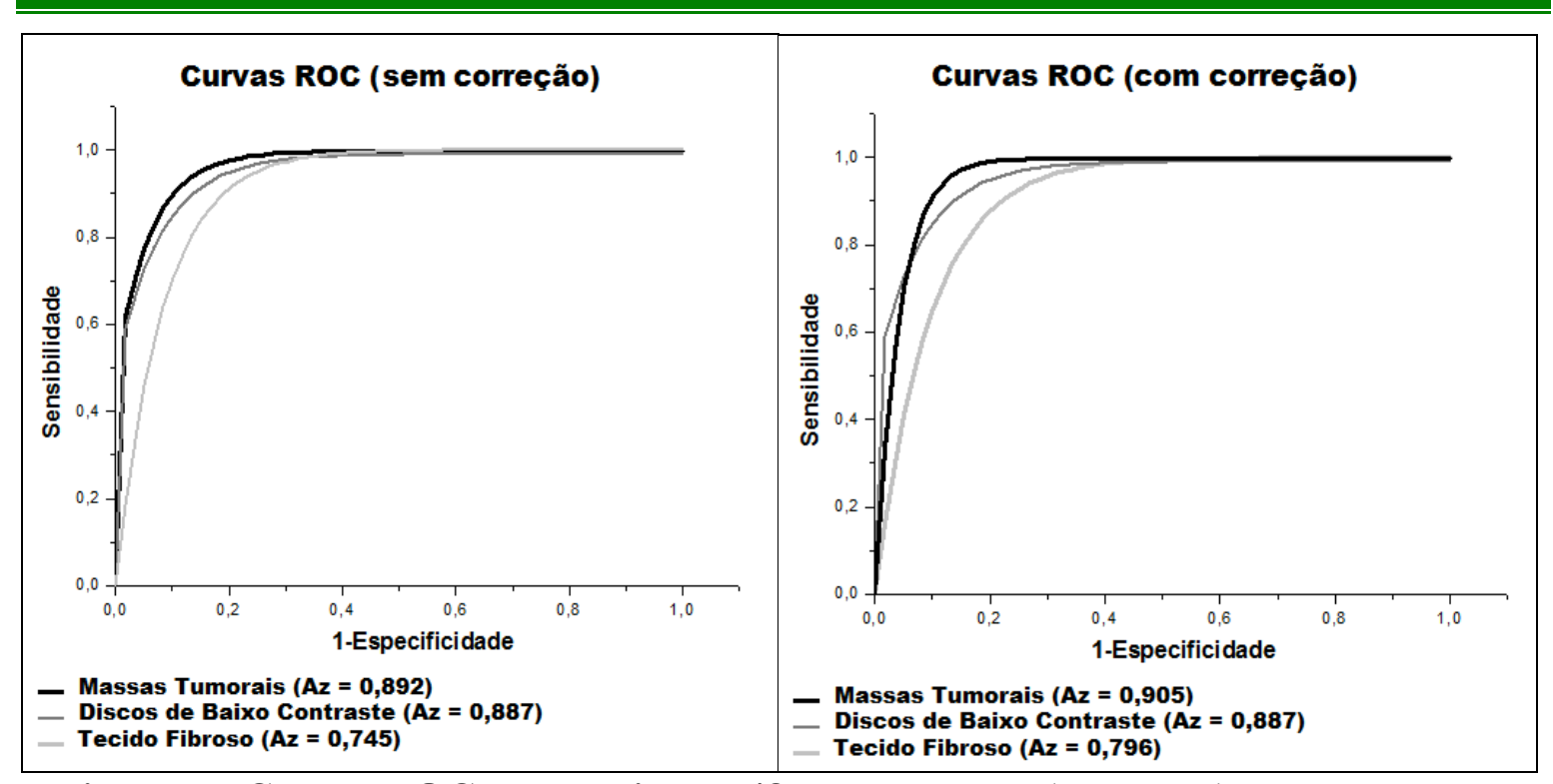

Figura 5 - Curvas ROC do algoritmo J48 sem correção (esquerda) e com correção (direita) para classificação do tecido fibroso, discos de baixo contraste e massas tumorais.

\section{DISCUSSÕES E CONCLUSÃO}

O processo de correção da digitalização das imagens do simulador radiográfico apresentou uma melhora na classificação das estruturas que compõem o tecido fibroso. Apesar de existir uma redução na taxa de sensibilidade dessas estruturas, houve um aumento em sua especificidade, o que indica uma melhor taxa de eficiência do método de correção. Acredita-se que por os discos de baixo contraste e massas tumorais apresentarem melhor definição com relação ao tecido fibroso, não houve uma melhora significativa no desempenho da técnica para a classificação.

Além disso, foi possível analisar a variação dos valores de pixels em diferentes posições do digitalizador. O método de correção realizado pelo o ajuste da curva característica do scanner em relação à curva do filme sensibilizado garante a uniformidade da digitalização em qualquer região do scanner avaliado (Microteck ScanMaker i800).

As estruturas que simulam as massas tumorais são as que apresentam um maior contraste em relação às outras estruturas analisadas. Por esse motivo, a classificação a respeito de sua visibilidade se torna mais fácil quando comparada com a das estruturas que representam os discos ou fibras.

Um dos problemas enfrentados é a presença de ruído que se assemelha a algumas estruturas. O tamanho reduzido das microcalcificações favorece a confusão entre estrutura e ruído, especialmente nos conjuntos menos evidentes. Estão sendo desenvolvidas técnicas para o tratamento de ruído dessas imagens para uma melhor detecção e classificação dessas estruturas.

Com os resultados apresentados, o método de correção das imagens do simulador radiográfico será inserido ao sistema Qualimamo com finalidade de melhorar a classificação das estruturas do tecido fibroso e, futuramente, microcalcificações. Após o término do trabalho, essa ferramenta de auxílio ao diagnóstico médico pode-se tornar 
viável para automação de parte dos procedimentos de controle de qualidade dos mamógrafos.

\section{AGRADECIMENTOS}

Ao CNPq, à CAPES e à SESU/MEC, pelo apoio na forma de bolsas de pesquisa e custeio.

\section{REFERÊNCIAS}

1. BARUFALDI B., SANTANA E.F., MARQUES J.R.T, MASCENA E.N., POEL J.V D., BATISTA L.V. "Sistema para o Controle de Qualidade de Mamógrafos e Geração Automática de Laudos Através da Análise de Imagens de Fantoma", V Workshop de Visão Computacional, São Paulo, 2009.

2. CHAN H.P., NIKLADSON L.T., IKEDA D.M., LAM K.L., ADLER D.D. "Digitization requirements in mammography: effects on computer-aided detection of microcalcifications". Medical Physics, v.21, n.7, p.1203-1211, 2004.

3. CINTI M.N. et al. "Custom Breast Phantom for an Accurate Tumor SNR Analysis", IEEE Transactions on Nuclear Science, vol. 51(1) 198-204, 2004.

4. GONZALES R.C., WOODS R.E. (2002) "Digital Image Processing", 2nd ed. Prentice Hall.

5. GONZALEZ-LOPEZ, A. "Useful optical density range in film dosimetry: Limitations due to noise and saturation" Phys. Med. Biol. 52, p.321-327, 2007.

6. HOGGE J. et al. "Quality Assurance in Mammography: Artifact Analysis", RadioGraphics, vol. 19 503-522, 1999.

7. INCA - Instituto Nacional do Câncer. "Estimativa 2010: incidência de câncer no Brasil / Instituto Nacional de Câncer”, ISBN 978-85-7318-161-6 (98), 2009.

8. MEDEIROS R.B., ALVES F.F.R., RUBERTI E.M, FERREIRA D.F.P "Influência das condições de processamento no desempenho de dois sistemas tela/filme utilizados na mamografia”, Revista Brasileira de Engenharia Biomédica, vol. 18 (2) 57-63, 2002.

9. TIEZZI D.G. "Câncer de mama: um futuro desafio para o sistema de saúde nos países em desenvolvimento"' Rev. Bras. Ginecol. Obstet. 32(6), 2010.

10. WITTEN I.H., FRANK E. "Data Mining - Practical Machine Learning Tools and Techniques". San Francisco, Elsevier, 2nd Edition, 2005. 